\title{
From Markovian semigroup to non-Markovian quantum evolution
}

\author{
Dariusz Chruściński and Andrzej Kossakowski \\ Institute of Physics, Nicolaus Copernicus University \\ Grudziadzka 5/7, 87-100 Toruń, Poland
}

\begin{abstract}
We provided a class of legitimate memory kernels leading to completely positive trace preserving dynamical maps. Our construction is based on a simple normalization procedure. Interestingly, when applied to the celebrated Wigner-Weisskopf theory it gives the standard Markovian evolution governed by the local master equation.
\end{abstract}

PACS numbers: 03.65.Yz, 03.65.Ta, 42.50.Lc

\section{INTRODUCTION}

The dynamics of open quantum systems attracts nowadays increasing attention [1-3]. It is relevant not only for the better understanding of quantum theory but it is fundamental in various modern applications of quantum mechanics. Since the system-environment interaction causes dissipation, decay and decoherence it is clear that dynamic of open systems is fundamental in modern quantum technologies, such as quantum communication, cryptography and computation [4].

The usual approach to the dynamics of an open quantum system consists in applying the Born-Markov approximation, that leads to the following master equation

$$
\frac{d}{d t} \rho_{t}=L \rho_{t}, \quad \rho_{0}=\rho,
$$

where $L$ denotes the corresponding Markovian generator (see Section III for all details). However, it turns out that description of many complex systems requires more sophisticated analysis which take into account nonMarkovian memory effects [1]. A popular non-Markovian generalization of (10) is the following nonlocal equation

$$
\dot{\rho}_{t}=\int_{0}^{t} \mathcal{K}_{t-\tau} \rho_{\tau} d \tau
$$

in which quantum memory effects are taken into account through the introduction of the memory kernel $\mathcal{K}_{\tau}$. It is clear from (2) that the rate of change of the state $\rho_{t}$ at time $t$ depends on its history (starting at $t=0$ ). The Markovian master equation (10) is reobtained when $\mathcal{K}_{\tau}=2 \delta(\tau) L$. The time dependent kernel $\mathcal{K}_{t}$ is usually referred to as the generator of the non-Markovian master equation.

Non-Markovian systems appear in many branches of physics, such as quantum optics [1, [5], solid state physics [6], quantum chemistry [7], and quantum information processing [8]. Since non-Markovian dynamics modifies exponential decay of quantum coherence it turns out that when applied to composite systems it may protect quantum entanglement for longer time than standard Markovian evolution [9]. In particular it may protect the system against the sudden death of entanglement [10]. NonMarkovian dynamics was recently studied in [11 23]. Interestingly, several measures of non-Markovianity were proposed during last year 24 27].
One of the fundamental problems in the theory of nonMarkovian master equations is to find those conditions on $\mathcal{K}_{t}$ that ensure that the time evolution $\Lambda_{t}$ defined by

$$
\rho \longrightarrow \rho_{t}=\Lambda_{t} \rho
$$

is completely positive and trace preserving $11,13,19$ 21]. This problem is very involved and contrary to the Markovian case the full characterization of the corresponding properties of memory kernel is still unknown.

In the present paper we provide a class of memory kernels giving rise to legitimate quantum dynamics. Our construction is based on a simple ide of normalization: starting from a family of completely positive maps satisfying a certain additional condition one is able to "normalize' it in order to obtain legitimate, i.e. trace preserving, quantum dynamics. As a result on obtains a class of legitimate memory kernels.

The paper is organized as follows. In Section vide the hierarchy of necessary conditions for the memory kernel which guarantee the legitimate quantum dynamics. Section III discusses the structure of Markovian semigroup and introduces basic idea of normalization. It turns out that Markovian semigroup appears as a normalized Wigner-Weisskopf theory. Section IV provides the main body of the paper. We show that there is a natural way to construct a legitimate memory kernel via an appropriate normalization procedure. Then in Section $[\mathrm{V}$ as a byproduct we provide the construction of legitimate memory kernels in classical stochastic nonMarkovian dynamics. In Section VI we analyze the reduction of the Schrödinger dynamics in the Hilbert space 'system + reservoir'. It turn out that reduced dynamics may be normalized to legitimate non-Markovian dynamics in the space of density operators. Final conclusions are collected in the last Section.

\section{MEMORY KERNELS AND QUANTUM BERNSTEIN THEOREM}

A solution $\Lambda_{t}$ to the non-Markovian master equation is trace preserving iff $\operatorname{Tr}\left(\mathcal{K}_{t} \rho\right)=0$ for any density operator $\rho$. Equivalently, this condition may be rewritten in terms of the dual of $\mathcal{K}_{t}$ as follows

$$
\mathcal{K}_{t}^{\#} \mathbb{I}=0,
$$


where $\mathbb{I}$ denotes an identity operator (recall that if $K$ is a linear map $K: \mathcal{B}(\mathcal{H}) \longrightarrow \mathcal{B}(\mathcal{H})$, then its dual $K^{\#}$ is defined by $\operatorname{Tr}\left(K^{\#} a \cdot b\right)=\operatorname{Tr}(a \cdot K b)$ for any $\left.a, b \in \mathcal{B}(\mathcal{H})\right)$.

Let us turn to more difficult part, i.e. complete positivity of $\Lambda_{t}$. Let us observe that taking the Laplace transform of (2) one obtains

$$
\widetilde{\Lambda}_{s}=\frac{1}{s-\widetilde{\mathcal{K}}_{s}},
$$

where

$$
\widetilde{\Lambda}_{s}=\int_{0}^{\infty} e^{-s t} \Lambda_{t} d t,
$$

and $s \in \mathbb{C}$. Now, if $\Lambda_{t}$ is completely positive then for $s>0$ its Laplace transform $\widetilde{\Lambda}_{s}$ is completely positive as well. Observe that

$$
(-1)^{k} \frac{d^{k}}{d s^{k}} \widetilde{\Lambda}_{s}=\int_{0}^{\infty} e^{-s t} t^{k} \Lambda_{t} d t .
$$

Now, the r.h.s of (7) is completely positive being a convex combination of completely positive maps $\Lambda_{t}$ (for $s>0$ ). Hence, using (5) one finds that for any positive integer $k$ and $s>0$

$$
(-1)^{k} \frac{d^{k}}{d s^{k}} \frac{1}{s-\widetilde{\mathcal{K}}_{s}} \quad \text { is completely positive . }
$$

This provides a series of necessary conditions for $\mathcal{K}_{t}$. Note, that these conditions may be considered as a quantum version of Bernstein theorem 28, 29]. Recall, that a function $f:[0, \infty) \rightarrow \mathbb{R}$ is completely monotone if

$$
(-1)^{k} \frac{d^{k}}{d t^{k}} f(t) \geq 0,
$$

for all $t \in[0, \infty)$ and $k=0,1,2, \ldots$ Then Bernstein theorem states that $f$ is completely monotone if and only if $f$ is a Laplace transform of the non-negative function, that is,

$$
f(s)=\int_{0}^{\infty} e^{-s t} g(t) d t,
$$

with $g(t) \geq 0$ and positive $s$. Unfortunately, apart from mathematical elegance the above infinite hierarchy of necessary conditions are very hard to use in practice. In particular if $\mathcal{K}_{t}$ is non-commutative family, that is, $\left[\mathcal{K}_{t}, \mathcal{K}_{\tau}\right] \neq 0$ for $t \neq \tau$, then even simple differentiation in (8) is by no means trivial. Therefore, one needs other tools to analyze properties of $\mathcal{K}_{t}$ which guarantee that $\Lambda_{t}$ is a legitimate quantum evolution with memory.

\section{THE STRUCTURE OF MARKOVIAN DYNAMICS}

To present our ideas we start with Markovian semigroup. As is well known [30, 31] the most general structure of the Markovian master equation is given by

$$
\frac{d}{d t} \rho_{t}=L \rho_{t}, \quad \rho_{0}=\rho,
$$

where the Markovian generator $L$ is given by

$$
L \rho=-i[H, \rho]+\frac{1}{2} \sum_{\alpha}\left(\left[V_{\alpha}, \rho V_{\alpha}^{\dagger}\right]+\left[V_{\alpha} \rho, V_{\alpha}^{\dagger}\right]\right) .
$$

In the above formula $H$ represents system Hamiltonian and $\left\{V_{\alpha}\right\}$ is the collection of arbitrary operators encoding the interaction between system and the environment. Let us observe that $L$ may be rewritten in the following form

$$
L=B-Z,
$$

where $B$ is a completely positive map defined by the following Kraus representation

$$
B \rho=\sum_{\alpha} V_{\alpha} \rho V_{\alpha}^{\dagger},
$$

and the super-operator $Z$ reads as follows

$$
Z \rho=-i\left(C \rho-\rho C^{\dagger}\right),
$$

with

$$
C=H-\frac{i}{2} B^{\#} \mathbb{I} .
$$

Note, that $B^{\#}$ denotes the dual map

$$
B^{\#} a=\sum_{\alpha} V_{\alpha}^{\dagger} a V_{\alpha},
$$

and hence $B^{\#} \mathbb{I}=\sum_{\alpha} V_{\alpha}^{\dagger} V_{\alpha}$ satisfies $B^{\#} \mathbb{I} \geq 0$. Note, that by construction

$$
B^{\#} \mathbb{I}=Z^{\#} \mathbb{I},
$$

which implies that $L^{\#} \mathbb{I}=0$, and hence the dynamics $\Lambda_{t}$ preserves the trace.

Now comes the natural question: which term in (13) is more fundamental $B$ or $Z$ ? Clearly, knowing completely positive $B$ the $Z$ part is up to the Hamiltonian part uniquely defined. On the other hand if $B=0$, then $Z \rho=-i[H, \rho]$ reduces to the purely Hamiltonian part. Hence, in our opinion, ' $Z$ part' plays a primary role replacing Hamiltonian $H$ by non-Hermitian operator $C$

$$
H \rightarrow C=H-\frac{i}{2} X,
$$

with $X \geq 0$, that is, one introduces non-Hermitian Hamiltonian $C$ giving rise to non-unitary dynamics. This approach is the heart of celebrated Wigner-Weisskopf theory [32]. Actually, in the standard Wigner-Weisskopf theory $C$ is normal $\left(C C^{\dagger}=C^{\dagger} C\right)$ which means that $[H, X]=0$. In this case there is an orthonormal basis $|k\rangle$ in $\mathcal{H}$ such that

$$
H|k\rangle=E_{k}|k\rangle, \quad X|k\rangle=\Gamma_{k}|k\rangle,
$$

with $\Gamma_{k} \geq 0$, for $k=1,2, \ldots, \operatorname{dim} \mathcal{H}$. Therefore, if $\left|\psi_{0}\right\rangle=$ $\sum_{k} c_{k}|k\rangle$, then

$$
\left|\psi_{t}\right\rangle=e^{-i C t}\left|\psi_{0}\right\rangle=\sum_{k} c_{k}(t)|k\rangle,
$$


with

$$
c_{k}(t)=e^{-\left(i E_{k}+\frac{1}{2} \Gamma_{k}\right) t} c_{k}
$$

and hence one recovers celebrated exponential decay. We stress, however, that $C$ needs not be normal. The only essential thing is that $X$ in (19) is positive semidefinite. Actually, there is big activity in the field of non-Hermitian Hamiltonians displaying real spectra (see e.g. recent review by Bender [33]).

Now, let $N_{t}$ be solution to

$$
\dot{N}_{t}=-Z N_{t}, \quad N_{0}=11 .
$$

One easily finds

$$
N_{t} \rho=e^{-i C t} \rho e^{i C^{\dagger} t} .
$$

It is evident that $N_{t}$ is completely positive. Note however that it does not preserve the trace (unless $X=0$ ). One finds

$$
N_{t}^{\#} \mathbb{I}=e^{-i C t} e^{i C^{\dagger} t}
$$

and if $C$ is normal it simplifies to $N_{t}^{\#} \mathbb{I}=e^{-X t}$. Interestingly,

$$
\dot{N}_{t}^{\#} \mathbb{I}=-e^{-i C t} X e^{i C^{\dagger} t}
$$

and hence

$$
-\dot{N}_{t}^{\#} \mathbb{I} \geq 0
$$

This condition would play a crucial role in our analysis of non-Markovian evolution. Here, we point out that it is trivially satisfied in the Markovian case.

It is, therefore, clear the ' $B$ term' is needed just to normalize evolution. Let us observe the the choice of completely positive $B$ is highly non unique. The only condition for $B$ is $B^{\#} \mathbb{I}=X$.

Finally, the Laplace transforms of (11) and (23) give

$$
\widetilde{\Lambda}_{s}=\frac{1}{s+Z-B}, \quad \widetilde{N}_{s}=\frac{1}{s+Z},
$$

and hence on obtains the following relation

$$
\widetilde{\Lambda}_{s}=\widetilde{N}_{s}+\widetilde{N}_{s} B \widetilde{\Lambda}_{s} .
$$

Iterating this equation yields the following perturbation series

$$
\widetilde{\Lambda}_{s}=\widetilde{N}_{s}+\tilde{N}_{s} B \tilde{N}_{s}+\tilde{N}_{s} B \widetilde{N}_{s} B \widetilde{N}_{s}+\ldots .
$$

Now, since both $\widetilde{N}_{s}$ and $B$ are completely positive it is clear from (30) that $\widetilde{\Lambda}_{s}$ is completely positive. Going back to the time-domain it finally shows that $\Lambda_{t}$ defines legitimate quantum evolution.

Note, that analyzing the series (30) one is tempted to relax the condition of complete positivity for $B$. It is evident that it is sufficient that $B \widetilde{N}_{t}$ is completely positive. Note, however, that due to (24) the map $N_{t}$ is invertible and the inverse

$$
N_{t}^{-1} \rho=e^{i C t} \rho e^{-i C^{\dagger} t}
$$

is completely positive as well. Hence $\left(B N_{t}\right) N_{t}^{-1}$ is again completely positive. But $\left(B N_{t}\right) N_{t}^{-1}=B$ which shows that complete positivity of $B$ cannot be relaxed.

\section{A CLASS OF LEGITIMATE MEMORY KERNELS}

In this section which provides the main body of the paper we generalize 'normalizing procedure' from semigroups to non-Markovian dynamics. We shall consider a class of non-Markovian Master Equations

$$
\frac{d}{d t} \Lambda_{t}=\int_{0}^{t} \mathcal{K}_{t-\tau} \Lambda_{\tau} d \tau, \quad \rho_{0}=\rho
$$

assuming that the memory kernel $\mathcal{K}_{t}$ - in analogy to (13) - can be represented in the following form

$$
\mathcal{K}_{t}=B_{t}-Z_{t}
$$

Unfortunately, we do not know how to chose $B_{t}$ and $Z_{t}$ in order to generate legitimate dynamical map $\Lambda_{t}$. Note that to satisfy (4), one has the following constraint

$$
B_{t}^{\#} \mathbb{I}=Z_{t}^{\#} \mathbb{I}
$$

The most difficult part is to guarantee that $\Lambda_{t}$ is completely positive for all $t \geq 0$.

To find legitimate $B_{t}$ and $Z_{t}$ we propose the following procedure: let us introduce an arbitrary map $N_{t}$ which is completely positive and satisfies initial condition $N_{0}=1$. Note, that $N_{t}$ may be represented as follows

$$
N_{t}=\mathbb{1}-\int_{0}^{t} F_{\tau} d \tau
$$

where $F_{t}=-\dot{N}_{t}$. Assuming that $N_{t}$ is differentiable it always satisfies the following non-local equation

$$
\frac{d}{d t} N_{t}=-\int_{0}^{t} Z_{t-\tau} N_{\tau} d \tau, \quad N_{0}=11
$$

where the corresponding generator $Z_{t}$ is defined in terms of its Laplace transform

$$
\widetilde{Z}_{s}=\frac{11-s \widetilde{N}_{s}}{\widetilde{N}_{s}}
$$

Hence, any family of completely positive maps $N_{t}$ provides $Z_{t}$ in (33). To provide $B_{t}$ satisfying condition (34) let us observe that equation (32) implies

$$
\widetilde{\Lambda}_{s}=\frac{1}{s+\widetilde{Z}_{s}-\widetilde{B}_{s}} .
$$

Moreover, one gets from (37)

$$
\widetilde{N}_{s}=\frac{1}{s+\widetilde{Z}_{s}}
$$

Hence

$$
\widetilde{\Lambda}_{s}=\widetilde{N}_{s}+\widetilde{N}_{s} \widetilde{B}_{s} \widetilde{\Lambda}_{s}
$$


Iterating this equation yields the following perturbation series

$$
\widetilde{\Lambda}_{s}=\widetilde{N}_{s}+\widetilde{N}_{s} \widetilde{B}_{s} \widetilde{N}_{s}+\widetilde{N}_{s} \widetilde{B}_{s} \widetilde{N}_{s} \widetilde{B}_{s} \widetilde{N}_{s}+\ldots
$$

This equation generalizes the Markovian formula (30). It is therefore clear that if the map $\widetilde{B}_{s} \widetilde{N}_{s}$, or equivalently in the time domain $\int_{0}^{t} B_{t-\tau} N_{\tau} d \tau$ is completely positive, then due to (41) the corresponding dynamical map $\Lambda_{t}$ is completely positive as well. Let us assume that the family of completely positive maps $N_{t}$ is invertible (clearly, $N_{t}^{-1}$ needs not be completely positive). Now, let us define $B_{t}$ is terms of its Laplace transform

$$
\widetilde{B}_{s}=\widetilde{Q}_{s} \widetilde{N}_{s}^{-1}
$$

where $\widetilde{Q}_{s}$ denotes the Laplace transform of the completely positive map $Q_{t}$. Equivalently, one has the following prescription for the Laplace transform of the memory kernel

$$
\widetilde{\mathcal{K}}_{s}=\widetilde{Q}_{s} \widetilde{N}_{s}^{-1}-\widetilde{Z}_{s}
$$

Now, $\widetilde{B}_{s} \widetilde{N}_{s}=\widetilde{Q}_{s}$ is by construction completely positive and hence due to (41) the above memory kernel generates completely positive dynamics. One obtains

$$
\widetilde{\mathcal{K}}_{s}=\left[\widetilde{Q}_{s}-\left(11-s \widetilde{N}_{s}\right)\right] \widetilde{N}_{s}^{-1},
$$

and hence

$$
\widetilde{\mathcal{K}}_{s}^{\#} \mathbb{I}=\widetilde{N}_{s}^{-1 \#}\left[\widetilde{Q}_{s}^{\#}-\left(\mathbb{1}-s \widetilde{N}_{s}^{\#}\right)\right] \mathbb{I}
$$

Now, if

$$
\left[\widetilde{Q}_{s}^{\#}-\left(\mathbb{1}-s \widetilde{N}_{s}^{\#}\right)\right] \mathbb{I}=0
$$

then $\widetilde{K}_{t}$ defines legitimate memory kernel. Recalling, that $1-s \widetilde{N}_{s}$ corresponds to the Laplace transform of $d N_{t} / d t$ one may rewrite (46) as follows

$$
Q_{t}^{\#} \mathbb{I}+\dot{N}_{t}^{\#} \mathbb{I}=0
$$

or, using 35

$$
Q_{t}^{\#} \mathbb{I}=F_{t}^{\#} \mathbb{I}
$$

Now, it is clear that if $F_{t}^{\#} \mathbb{I} \geq 0$ for all $t \geq 0$, then one can always find completely positive $Q_{t}$ satisfying (48).

Summarizing, we proved the following

Theorem 1 Let $N_{t}$ be an arbitrary (differentiable) family of completely positive maps satisfying (35) such that $F_{t}^{\#} \mathbb{I} \geq 0$. Then, there exists a family of completely positive maps $Q_{t}$ satisfying (48) and the formula (44) defines the Laplace transform of the legitimate generator of nonMarkovian dynamical map.
It should be clear that the construction of $Q_{t}$ is highly non unique. If $F_{t}^{\#} \mathbb{I} \geq 0$, then there is a time-dependent family of operators $M_{\alpha}(t)$ such that

$$
F_{t}^{\#} \mathbb{I}=\sum_{\alpha} M_{\alpha}^{\dagger}(t) M_{\alpha}(t)
$$

and hence one can define $Q_{t}$ via the following Kraus representation

$$
Q_{t} \rho=\sum_{\alpha} M_{\alpha}(t) \rho M_{\alpha}^{\dagger}(t)
$$

Again, the choice of $M_{\alpha}(t)$ is highly non unique. Note, that the simplest way to satisfy (48) is to take

$$
Q_{t}=B F_{t},
$$

where $B$ denotes a quantum channel (completely positive trace preserving map). Indeed, one has $Q_{t}^{\#} \mathbb{I}=$ $F_{t}^{\#}\left(B^{\#} \mathbb{I}\right)=F_{t}^{\#} \mathbb{I}$, due to $B^{\#} \mathbb{I}=\mathbb{I}$. In this case one obtains the following form of the memory kernel

$$
\mathcal{K}_{t}=(B-\mathbb{1}) Z_{t}
$$

Example 1 Let us observe that $Q_{t}$ defined via (51) is completely positive whenever $F_{t}$ is completely positive. Note, however, that due to (35) the map $F_{t}$ can not be completely positive unless $F_{t}=f(t) 11$ for some nonnegative function $f$. Indeed, the corresponding Choi matrices for $N_{t}, F_{t}$ and identity map 11 have to be positive. Now, the Choi matrix for the identity map defines rank-1 projector $P^{+}$(the maximally entangled state in $\mathcal{H} \otimes \mathcal{H}$ ) and therefore it is clear that we can not subtract from $\mathrm{P}^{+}$any positive operator unless it is proportional to $\mathrm{P}^{+}$ itself (otherwise the Choi matrix for $N_{t}$ would no longer be positive). Hence

$$
N_{t}=\left(1-\int_{0}^{t} f(\tau) d \tau\right) \mathbb{1},
$$

where $f(t) \geq 0$, and to guarantee complete positivity of $N_{t}$ one has

$$
\int_{0}^{\infty} f(t) d t \leq 1 .
$$

Finally, using (37) one finds the following formula

$$
Z_{t}=\kappa(t) \mathbb{1},
$$

where the function $\kappa(t)$ is defined in terms of its Laplace transform

$$
\widetilde{\kappa}(s)=\frac{s \tilde{f}(s)}{1-\widetilde{f}(s)} .
$$

To find $B_{t}$ we define its Laplace transform by $\widetilde{B}_{s}=$ $Q_{s} \widetilde{N}_{s}^{-1}$, where $Q_{t}$ are arbitrary completely positive maps. One has

$$
\tilde{N}_{s}^{-1}=\frac{s}{1-\widetilde{f}(s)} 1,
$$


and hence

$$
\widetilde{K}_{s}=\frac{s}{1-\widetilde{f}(s)}\left[\widetilde{Q}_{s}-\widetilde{f}(s) \mathbb{1}\right] .
$$

Note, that to satisfy (4) one has

$$
\widetilde{Q}_{s}^{\#} \mathbb{I}=\widetilde{f}(s) \mathbb{I} .
$$

In particular if $Q_{t}=B F_{t}$, then $\widetilde{Q}_{s}=\widetilde{f}(s) B$ and the memory kernel has the following form

$$
\mathcal{K}_{t}=\kappa(t)(B-\mathbb{1})
$$

with $B$ being an arbitrary quantum channel.

Example 2 Consider now the following class of completely positive maps $N_{t}$ in $\mathcal{B}(\mathcal{H})$ with $\mathcal{H}=\mathbb{C}^{d}$

$$
N_{t} \rho=\sum_{i, j=1}^{d} n_{i j}(t)|i\rangle\langle i|\rho| j\rangle\langle j|,
$$

where the matrix $\left[n_{i j}(t)\right] \geq 0$ for $t \geq 0$, and $n_{i j}(0)=1$ which guaranties that $N_{0}=1$. Equation (35) implies the following formula for the corresponding map $F_{t}$

$$
F_{t} \rho=\sum_{i, j=1}^{d} f_{i j}(t)|i\rangle\langle i|\rho| j\rangle\langle j|,
$$

where

$$
f_{i j}(t)=-\frac{d n_{i j}(t)}{d t} .
$$

Let $Q_{t}$ be a family of complete positive maps defined by the corresponding Kraus representation

$$
Q_{t} \rho=\sum_{i, j, k, l=1}^{d} q_{i j ; k l}(t)|i\rangle\langle j|\rho| l\rangle\langle k| .
$$

It is clear that $Q_{t}$ is completely positive iff

$$
\sum_{i, j, k, l=1}^{d} q_{i j ; k l}(t) x_{i j} \overline{x_{k l}} \geq 0
$$

for any $d \times d$ complex matrix $\left[x_{i j}\right]$. One has

$$
F_{t}^{\#} \mathbb{I}=\sum_{k} f_{k k}(t)|k\rangle\langle k|
$$

and hence $F_{t}^{\#} \mathbb{I} \geq 0$ if $f_{k k}(t)=-\dot{n}_{k k}(t) \geq 0$. One finds for $Q_{t}^{\#} \mathbb{I}$

$$
Q_{t}^{\#} \mathbb{I}=\sum_{i, j, k} q_{i j ; k j}(t)|k\rangle\langle i| .
$$

Now, to satisfy (48) one has

$$
q_{i j ; k l}(t)=\delta_{i k} c_{j l}^{(k)}(t)
$$

where the time-dependent $d \times d$ complex matrices $c^{(k)}(t)$ are positive semi-definite, i.e. they define unnormalized density operators. Finally, to satisfy (48) one has the following conditions

$$
\operatorname{Tr} c^{(k)}(t)=f_{k k}(t),
$$

for $k=1,2, \ldots, d$.

One may ask how to construct (61) in order to satisfy $\dot{n}_{k k}(t) \leq 0$. Here we present the following construction: let $X_{1}, \ldots, X_{d}$ be a set of arbitrary linear operators from $\mathcal{B}(\mathcal{H})$. Define

$$
n_{i j}(t)=\operatorname{Tr}\left(\omega e^{t X_{i}^{\dagger}} e^{t X_{j}}\right)
$$

where $\omega$ is a fixed density operator. By construction $\left[n_{i j}(t)\right] \geq 0$. Moreover, $n_{i j}(0)=\operatorname{Tr} \omega=1$. One obtains

$$
f_{i j}(t)=-\dot{n}_{i j}(t)=-\operatorname{Tr}\left(\omega e^{t X_{i}^{\dagger}}\left(X_{i}^{\dagger}+X_{j}\right) e^{t X_{j}}\right)
$$

and hence

$$
f_{i i}(t)=-\operatorname{Tr}\left(\omega_{i}\left(X_{i}^{\dagger}+X_{i}\right)\right)
$$

where

$$
\omega_{i}=e^{t X_{i}} \omega e^{t X_{i}^{\dagger}}
$$

Now, if each $X_{i}$ is dissipative, i.e. $X_{i}+X_{i}^{\dagger} \leq 0$, then one gets $f_{i i}(t) \geq 0$.

Remark 1 Let us observe hat there is another way to normalize the family of completely positive maps $N_{t}$. Suppose that $X_{t}:=N_{t}^{\#} \mathbb{I}>0$, for all $t \geq 0$ and define

$$
M_{t}^{\#} a=X_{t}^{-1 / 2}\left(N_{t}^{\#} a\right) X_{t}^{-1 / 2} .
$$

One has $M_{t}^{\#} \mathbb{I}=\mathbb{I}$, and hence $M_{t}$ defines a legitimate dynamical map. Note, however, that we are not able to write down the corresponding equation for $M_{t}$. Moreover, the above normalization is again highly non unique. If $U_{t}$ is an arbitrary family of unitary operators, then $a \rightarrow U_{t}\left(M_{t}^{\#} a\right) U_{t}^{\dagger}$ does preserve $\mathbb{I}$.

\section{CLASSICAL NON-MARKOVIAN DYNAMICS}

As a byproduct of our general approach one obtains a coherent description of classical stochastic dynamics. A mixed state of a $d$-state classical system is described by a stochastic $d$-vector $\left(p_{1}, \ldots, p_{d}\right)$. Any such vector may be encoded into diagonal density operator $\rho_{k l}=p_{k} \delta_{k l}$. We call a linear map in $\mathcal{B}(\mathcal{H})$ to be classical if it maps diagonal matrices into diagonal matrices (in a fixed basis in $\mathcal{H}$ ).

Consider now a classical completely positive maps

$$
N_{t} \rho=\sum_{i, j=1}^{d} n_{i}(t)|i\rangle\langle i|\rho| i\rangle\langle i|
$$


which is nothing but the 'diagonal part' of 61. Moreover, one assumes that $n_{i}(t) \geq 0$ and $n_{i}(0)=1$. Applying to probability vector the action of $N_{t}$ is very simple: it maps $p_{k}$ into $n_{k}(t) p_{k}$. Clearly, $N_{t}$ is not normalized: $N_{t}^{\#} \mathbb{I}=\sum_{k} n_{k}(t)|k\rangle\langle k|$. Moreover, it defines $Z_{t}$

$$
Z_{t} \rho=\sum_{i, j=1}^{d} z_{i}(t)|i\rangle\langle i|\rho| i\rangle\langle i|,
$$

where $z_{i}(t)$ are defined in terms of the Laplace transform

$$
\widetilde{z}_{i}(s)=\frac{1-s \widetilde{n}_{i}(s)}{\widetilde{n}_{i}(s)} .
$$

Now, let $Q_{t}$ be another family of classical completely positive maps

$$
Q_{t} \rho=\sum_{i, j=1}^{d} q_{i j}(t)|i\rangle\langle j|\rho| j\rangle\langle i|,
$$

where the time-dependent coefficients satisfy $q_{i j}(t) \geq 0$ for $t \geq 0$. Normalization condition (47) implies

$$
\sum_{i=1}^{d} q_{i j}(t)+\dot{n}_{j}(t)=0
$$

for $j=1, \ldots, d$. Let us introduce $B_{t}$ according to $\widetilde{B}_{s}=$ $\widetilde{Q}_{s} \widetilde{N}_{s}^{-1}$. One has

$$
B_{t} \rho=\sum_{i, j=1}^{d} b_{i j}(t)|i\rangle\langle j|\rho| j\rangle\langle i|,
$$

where $b_{i j}(t)$ are defined in terms of the Laplace transform

$$
\widetilde{b}_{i j}(s)=\frac{\widetilde{q}_{i j}(s)}{\widetilde{n}_{j}(s)} .
$$

Finally, one arrives to the following formula for the memory kernel

$$
\mathcal{K}_{t} \rho=\sum_{i, j=1}^{d} k_{i j}(t)|i\rangle\langle j|\rho| j\rangle\langle i|,
$$

where $k_{i j}(t)$ are defined as follows

$$
k_{i j}(t)=b_{i j}(t)-\delta_{i j} z_{j}(t) .
$$

Observe, that (79) implies $\sum_{i=1}^{d} k_{i j}(t)=0$ and hence

$$
\sum_{i=1}^{d} b_{i j}(t)=z_{j}(t) .
$$

When translated into the stochastic vector our approach gives rise to the following classical non-Markovian master equation

$$
\dot{p}_{i}(t)=\sum_{j=1}^{d} \int_{0}^{t} d \tau\left[b_{i j}(t-\tau) p_{j}(\tau)-b_{j i}(t-\tau) p_{i}(\tau)\right] .
$$

Let us consider the special case corresponding to (51). One introduces $B$ by

$$
B \rho=\sum_{i, j=1}^{d} \pi_{i j}|i\rangle\langle j|\rho| j\rangle\langle i|,
$$

where $\left[\pi_{i j}\right]$ is a stochastic matrix, i.e. $\pi_{i j} \geq 0$, and $\sum_{i} \pi_{i j}=1$. One finds

$$
Q_{t} \rho=\sum_{i, j=1}^{d} \pi_{i j} f_{j}(t)|i\rangle\langle j|\rho| j\rangle\langle i|
$$

where $f_{j}(t)=-\dot{n}_{j}(t)$, that is, $n_{j}(t)$ may be represented via

$$
n_{j}(t)=1-\int_{0}^{t} f_{j}(\tau) d \tau
$$

In this case

$$
b_{i j}(t)=\pi_{i j} \kappa_{j}(t),
$$

where $\kappa_{j}(t)$ are defined in terms of the Laplace transform

$$
\widetilde{\kappa}_{j}(s)=\frac{s \widetilde{f}_{j}(s)}{1-\widetilde{f}_{j}(s)} .
$$

Inserting into (85) one recovers the old result of Gillespie 34] (see also 35] and the discussion in 21] on continuoustime random walk).

\section{REDUCING SCHRÖDINGER DYNAMICS}

In this section we provide a simple construction giving rise to the family of completely positive maps $N_{t}$ satisfying initial condition $N_{0}=1$. Consider the unitary evolution in $\mathcal{H}_{S} \otimes \mathcal{H}_{R}$ governed by the Hamiltonian $H$. Let $|\omega\rangle \in \mathcal{H}_{R}$ be a fixed vector state of the reservoir and let us define the projector

$$
P: \mathcal{H}_{S} \otimes \mathcal{H}_{R} \longrightarrow \mathcal{H}_{S} \otimes \mathcal{H}_{R}
$$

by the following formula

$$
P=\mathbb{I}_{S} \otimes|\omega\rangle\langle\omega|
$$

that is,

$$
P(|\psi\rangle \otimes|\phi\rangle)=|\psi\rangle \otimes\langle\omega \mid \phi\rangle|\omega\rangle .
$$

Having defined $P$ one introduces the reduced dynamics in $\mathcal{H}_{S}$ by

$$
\left|\psi_{t}\right\rangle=n_{t}\left|\psi_{0}\right\rangle
$$

where the time-dependent evolution operators $n_{t}: \mathcal{H}_{S} \rightarrow$ $\mathcal{H}_{S}$ is defined by

$$
n_{t} \otimes|\omega\rangle\langle\omega|=P e^{-i t H} P,
$$


and satisfies $n_{0}=\mathbb{I}_{S}$. In analogy to 35 it can be represented as follows

$$
n_{t}=\mathbb{I}_{S}-\int_{0}^{t} \nu_{\tau} d \tau
$$

where $\nu_{t}=-\dot{n}_{t}$ and hence it satisfies non-local equation

$$
\dot{n}_{t}=-\int_{0}^{t} z_{t-\tau} n_{\tau} d \tau
$$

where the generator is defined in terms of its Laplace transform

$$
\widetilde{z}_{s}=\frac{\mathbb{I}_{S}-s \widetilde{n}_{s}}{\widetilde{n}_{s}}
$$

Equivalently, if $\left|\varphi_{t}\right\rangle$ is a solution of the Schrödinger equation

$$
i \frac{d\left|\varphi_{t}\right\rangle}{d t}=H\left|\varphi_{t}\right\rangle
$$

with an initial condition $\left|\varphi_{0}\right\rangle=\left|\psi_{0}\right\rangle \otimes|\omega\rangle$, then $\left|\psi_{t}\right\rangle$ is nothing but the reduction of $\left|\varphi_{t}\right\rangle$

$$
\left|\psi_{t}\right\rangle \otimes|\omega\rangle=P\left|\varphi_{t}\right\rangle
$$

It should be clear from (97) that the reduced evolution $n_{t}$ does not satisfy Schrödinger-like equation in $\mathcal{H}_{S}$. Note, that $n_{t}$ is a contraction in $\mathcal{H}_{S}$, that is

$$
\left\langle n_{t} \psi \mid n_{t} \psi\right\rangle \leq\langle\psi \mid \psi\rangle
$$

for $t \geq 0$, and hence $n_{t}$ does not define a legitimate dynamics of the pure state $\left|\psi_{t}\right\rangle$ in the system Hilbert space $\mathcal{H}_{S}$. One may easily define normalized solution

$$
\left|\psi_{t}^{\prime}\right\rangle=\frac{\left|\psi_{t}\right\rangle}{\left\|\psi_{t}\right\|}
$$

Note, however, that normalized evolution $\left|\psi_{t}^{\prime}\right\rangle$ is, contrary to $\left|\psi_{t}\right\rangle$, no longer linear.

Here, we follow our general approach. Let us define the following evolution in the space of mixed states

$$
N_{t} \rho=n_{t} \rho n_{t}^{\dagger}
$$

By construction $N_{t}$ is completely positive and satisfies an initial condition $N_{0}=11$. Interestingly, the inverse

$$
N_{t}^{-1}=n_{-t} \rho n_{-t}^{\dagger}
$$

does exist for almost all $t \geq 0$ and it is again completely positive. Hence, if

$$
-\dot{N}_{t}^{\#} \mathbb{I}=n_{t}^{\dagger} \nu_{t}+\nu_{t}^{\dagger} n_{t} \geq 0
$$

then one can find a family of completely positive maps $Q_{t}$ and define the legitimate memory kernel $\mathcal{K}_{t}=B_{t}-Z_{t}$.
Remark 2 Usually, $H=H_{0}+\lambda H_{\text {int }}$. It is well known [36] that in the weak coupling limit $\left|\psi_{t}\right\rangle=n_{t}\left|\psi_{0}\right\rangle$ satisfies satisfies the following equation

$$
\dot{n}_{t}=-z n_{t}, \quad n_{0}=11
$$

with $z=i h+\frac{1}{2} X$, where $h$ is Hermitian and $X \geq 0$ (hence Hermitian). Clearly, $z$ plays a role of Wigner-Weisskopf non-Hermitian Hamiltonian. Interestingly weak coupling limit guaranties that $z$ is a normal operator, i.e. $h$ and $X$ mutually commute.

Example 3 Let $n_{t}$ be defined by its spectral decomposition

$$
n_{t}|k\rangle=x_{k}(t)|k\rangle
$$

and hence one obtains

$$
N_{t} \rho=\sum_{k, l} n_{k l}(t)|k\rangle\langle k|\rho| l\rangle\langle l|
$$

where

$$
n_{k l}(t)=x_{k}(t) \overline{x_{l}(t)} \text {. }
$$

One finds

$$
N_{t}^{\#} \mathbb{I}=\sum_{k}\left|x_{k}(t)\right|^{2}|k\rangle\langle k|
$$

which shows that $N_{t}$ is normalized iff $x_{k}(t)=e^{-i \varepsilon_{k} t}$. Consider now

$$
x_{k}(t)=e^{-i \varepsilon_{k}}\left(1-\int_{0}^{t} f_{k}(\tau) d \tau\right) .
$$

Note, that if $f_{k}(t)$ has a special form

$$
f_{k}(t)=\kappa_{k} e^{-\gamma_{k} t}, \quad \gamma_{k} \geq 0,
$$

then

$$
x_{k}(t)=\gamma_{k}^{-1} e^{-i \varepsilon_{k}}\left(\gamma_{k}-\kappa_{k}+\kappa_{k} e^{-\gamma_{k} t}\right),
$$

and hence in the limit $\kappa_{k} \rightarrow \gamma_{k}$ one recovers WignerWeisskopf theory

$$
x_{k}(t)=e^{-\left[i \varepsilon_{k}+\gamma_{k}\right] t} .
$$

Example 4 Consider the pure decoherence model,

$$
H=H_{R}+H_{S}+H_{S R}
$$

where $H_{R}$ is the reservoir Hamiltonian,

$$
H_{S}=\sum_{k} \epsilon_{k} P_{k},
$$

with $P_{k}=|k\rangle\langle k|$, the system Hamiltonian and

$$
H_{S R}=\sum_{k} P_{k} \otimes B_{k}
$$


the interaction part, $B_{k}=B_{k}^{\dagger}$ being reservoirs operators. The total Hamiltonian has therefore the following form

$$
H=\sum_{k} P_{k} \otimes R_{k},
$$

where the reservoir operators $R_{k}$ read as follows

$$
R_{k}=\varepsilon_{k} \mathbb{I}_{R}+H_{R}+B_{k} .
$$

One easily finds the the reduced dynamics $n_{t}$ is defined by the formula (107) with

$$
x_{k}(t)=\left\langle\omega\left|e^{-i R_{k} t}\right| \omega\right\rangle=e^{-i \varepsilon_{k} t}\left\langle\omega\left|e^{-i\left[H_{R}+B_{k}\right] t}\right| \omega\right\rangle .
$$

The presence of nontrivial factor $\left\langle\omega\left|e^{-i\left[H_{R}+B_{k}\right] t}\right| \omega\right\rangle$ is responsible for all memory effects.

\section{CONCLUSIONS}

We have provided a class of legitimate memory kernels leading to completely positive trace preserving dynami- cal maps. Our construction is based on the simple observation that if the family of completely positive maps $N_{t}$ with an initial condition $N_{0}=11$ satisfies an additional condition $\dot{N}_{t}^{\#} \mathbb{I} \leq 0$, then one may perform suitable normalization and as a result one obtains a family of completely positive trace preserving maps $\Lambda_{t}$ generated by the legitimate memory kernel $\mathcal{K}_{t}$. This procedure is highly non unique. Interestingly, when applied to Wigner-Weisskopf theory it gives the standard Markovian evolution governed by the local master equation. As a byproduct we have constructed a class of legitimate memory kernels for classical stochastic non-Markovian dynamics.

\section{Acknowledgment}

This work was partially supported by the Polish Ministry of Science and Higher Education Grant No 3004/B/H03/2007/33.

[1] H.-P. Breuer and F. Petruccione, The Theory of Open Quantum Systems (Oxford Univ. Press, Oxford, 2007).

[2] U. Weiss, Quantum Dissipative Systems, (World Scientific, Singapore, 2000).

[3] R. Alicki and K. Lendi, Quantum Dynamical Semigroups and Applications (Springer, Berlin, 1987).

[4] M. A. Nielsen and I. L. Chuang, Quantum Computation and Quantum Information (Cambridge Univ. Press, Cambridge, 2000).

[5] C.W. Gardiner and P. Zoller, Quantum Noice, SpringerVerlag, Berlin, 1999.

[6] C. W. Lai, P. Maletinsky, A. Badolato, and A. Imamoglu, Phys. Rev. Lett. 96, 167403 (2006).

[7] M. B. Plenio and P. L. Knight, Rev. Mod. Phys. 70, 101 (1998).

[8] D. Aharonov, A. Kitaev, and J. Preskill, Phys. Rev. Lett. 96, 050504 (2006).

[9] D. Chruściński, A. Kossakowski, and S. Pascazio, Phys. Rev. A 81, 032101 (2010).

[10] T. Yu and J. H. Eberly, Opt.Commun. 264, 393 (2006); Quantum Inf. Comput. 7, 459 (2007); Phys. Rev. Lett. 97, 140403 (2006).

[11] J. Wilkie, Phys. Rev. E 62, 8808 (2000); J. Wilkie and Yin Mei Wong, J. Phys. A: Math. Theor. 42, 015006 (2009).

[12] A. A. Budini, Phys. Rev. A 69, 042107 (2004); ibid. 74, 053815 (2006).

[13] H.-P. Breuer, Phys. Rev. A 69022115 (2004); ibid. 70, 012106 (2004).

[14] S. Daffer, K. Wódkiewicz, J.D. Cresser, and J.K. McIver, Phys. Rev. A 70, 010304 (2004).

[15] A. Shabani and D.A. Lidar, Phys. Rev. A 71, 020101(R) (2005).

[16] S. Maniscalco, Phys. Rev. A 72, 024103 (2005).

[17] S. Maniscalco and F. Petruccione, Phys. Rev. A 73,

012111 (2006).

[18] J. Piilo, K. Härkönen, S. Maniscalco, K.-A. Suominen, Phys. Rev. Lett. 100, 180402 (2008); Phys. Rev. A 79, 062112 (2009).

[19] A. Kossakowski and R. Rebolledo, Open Syst. Inf. Dyn. 14, 265 (2007); ibid. 15, 135 (2008).

[20] A. Kossakowski and R. Rebolledo, Open Syst. Inf. Dyn. 16, 259 (2009).

[21] H.-P. Breuer and B. Vacchini, Phys. Rev. Lett. 101 (2008) 140402; Phys. Rev. E 79, 041147 (2009).

[22] M. Moodley and F. Petruccione, Phys. Rev. A 79, 042103 (2009).

[23] D. Chruściński and A. Kossakowski, Phys. Rev. Lett. 104, 070406 (2010).

[24] M.M. Wolf, J. Eisert, T.S. Cubitt, and J.I. Cirac, Phys. Rev. Lett. 101, 150402 (2008).

[25] H.-P. Breuer, E.-M. Laine, J. Piilo, Phys. Rev. Lett. 103, 210401 (2009).

[26] Á. Rivas, S.F. Huelga, and M.B. Plenio, Phys. Rev. Lett. 105, 050403 (2010).

[27] Xiao-Ming Lu, Xiaoguang Wang, and C.P. Sun, Phys. Rev. A 82, 042103 (2010).

[28] S.N. Bernstein, Acta Mathematica 52, 1 (1928).

[29] D. Widder, The Laplace Transform, Princeton University Press (1941).

[30] V. Gorini, A. Kossakowski, and E. C. G. Sudarshan, J. Math. Phys. 17, 821 (1976).

[31] G. Lindblad, Comm. Math. Phys. 48, 119 (1976).

[32] V. Weisskopf and E. Wigner, Z. Phys. 63 (1930) 54; ibid. 65 (1930) 18.

[33] C. M. Bender, Rep. Prog. Phys. 70, 947 (2007).

[34] D.T. Gillespie, Phys. Lett. A 64, 22 (1977).

[35] J. Klafter and R. Silbey, Phys. Rev. Lett. 44, 55 (1980).

[36] E. Davies, J. Math. Phys. 15, 2036 (1974). 